\title{
Enhanced Optical Emission from the Crab Pulsar
}

\author{
B. W. Stappers
}

Stichting ASTRON, 7990 AA Dwingeloo, The Netherlands

\author{
A. Shearer \\ National University of Ireland, Galway, Newcastle Road, Galway, \\ Ireland
}

\begin{abstract}
We have detected an enhancement in the optical emission from the Crab pulsar which is correlated with the presence of the giant radio pulses. Coincident optical pulses were found to be on average $3 \%$ brighter than those coincident with normal radio pulses. This enhancement, combined with the lack of any other pulse profile changes, indicates that both the giant radio pulses and the increased optical emission are most likely linked to an increase in the electron-positron plasma density.
\end{abstract}

\section{Introduction}

While some good progress has been made on our understanding of the pulsar emission mechanism (see for example contributions in this proceedings by Spitkovsky and by Melrose) its exact nature remains a matter of some debate. A broad consensus has emerged: the luminosity is powered by the slowing down of the rotation rate of the pulsar, the pulsed radio signal is generated via a coherent process, the emission in the optical-to-X-ray range is incoherent synchrotron radiation, and the $\gamma$-ray emission is curvature radiation. What is not agreed upon is the mechanism that accelerates the electrons to the energy required for synchrotron and curvature radiation, where this acceleration takes place, how coherency is maintained, and the stability of the electron-positron plasma outflow from the neutron star's surface (Harding \& Daugherty 1998; Romani 1996).

The Crab pulsar is one of the few pulsars which emits across all wavelengths; the higher-energy emission is seen to correspond with the two main radio peaks, whilst a smaller radio precursor has no high-energy counterpart (Fig. 1). One suggestion is that the precursor represents emission from the pulsar polar cap region near the neutron star surface, similar to the radio emission from most pulsars, and that the other features come from higher in the magnetosphere (Romani \& Yadigaroglu 1995).

The Crab pulsar also belongs to the even smaller class of pulsars which exhibit so-called giant radio pulses (GRPs). These GRPs occur at random intervals, in phase with either the main pulse or interpulse (Fig. 1), and have energies up to 10000 times larger than the normal pulses (Lundgren et al. 1995). A link between these GRPs and the high energy emission might be expected, 


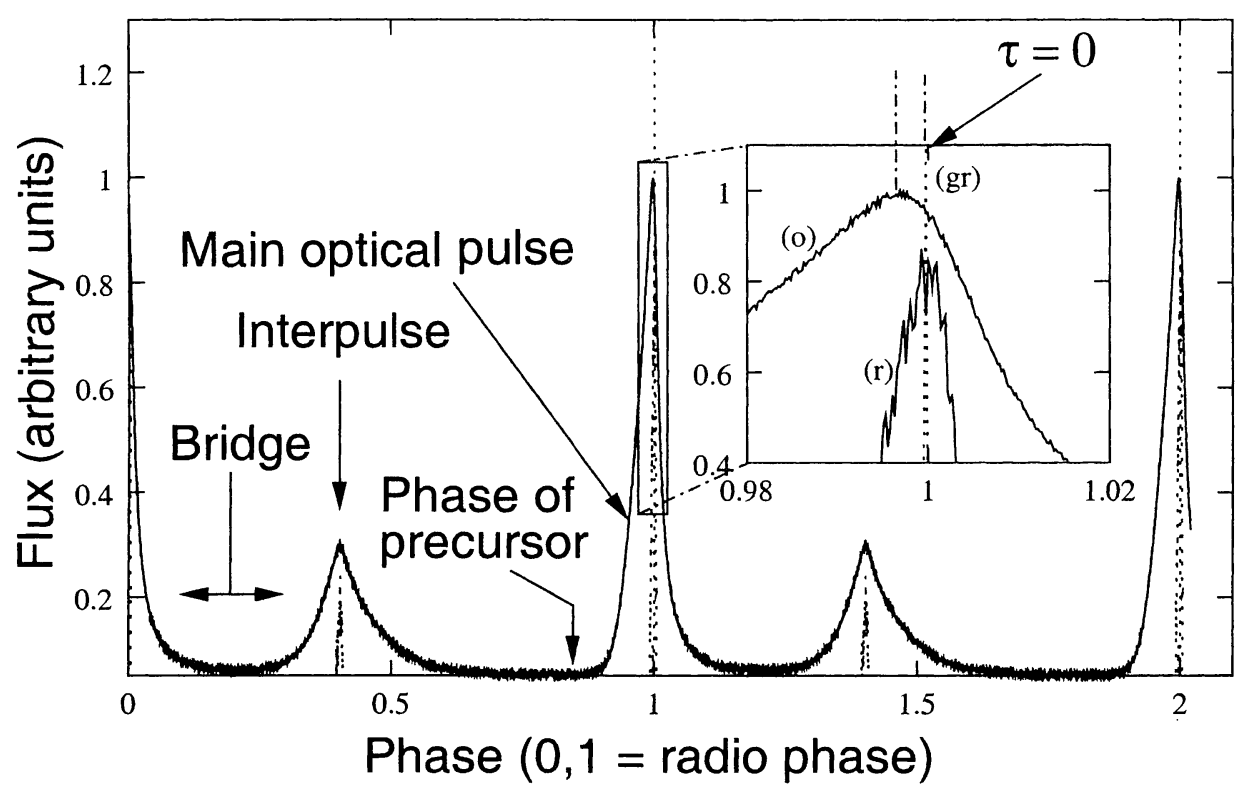

Figure 1. The Crab pulse profile showing the optical light curve (o), the average radio light curve at $1380 \mathrm{MHz}(\mathrm{r})$ and a single giant pulse at $1357.5 \mathrm{MHz}(\mathrm{gr})$. The optical light curve is taken from the second night of observation.

since the only pulsars discovered so far to exhibit GRPs have high values for the magnetic field strength at the light cylinder, a location where some models have the high-energy emission originating. A connection between these two emission processes can be used to probe the relative role of coherence variability versus pair-creation fluctuations in creating GRPs. A previous study found that the $\gamma$-ray emission associated with GRPs showed no evidence for enhancement at 2.5 times the average (Lundgren et al. 1995). This study was limited by low statistics, but as the Crab pulsar is a bright optical source, we decided to search for a correlation there. Previous work has shown that in the optical and infrared energy regions, the pulse profile is constant at the $1 \%$ level (Hegyi, Novick \& Thaddeus 1971; Jones, Smith \& Nelson 1980). However these studies did not search for a correlation with GRPs and instead considered all pulses.

\section{Observations, Data Reduction and Results}

Our simultaneous observations were carried out over two nights using the 4.2$\mathrm{m}$ William Herschel Telescope and the Westerbork Synthesis Radio Telescope. Optical data were recorded using the high speed optical photometer TRIFFID (Buckton et al. 2003) with a time resolution of $12.5 \mu \mathrm{s}$, while the $5-\mathrm{MHz}$ radio band was recorded using the baseband mode of PuMa (Voûte et al. 2002) and subsequently coherently dedispersed and smoothed to a time resolution of $6.4 \mu \mathrm{s}$. The data were time aligned at the solar system barycenter using the Jodrell 


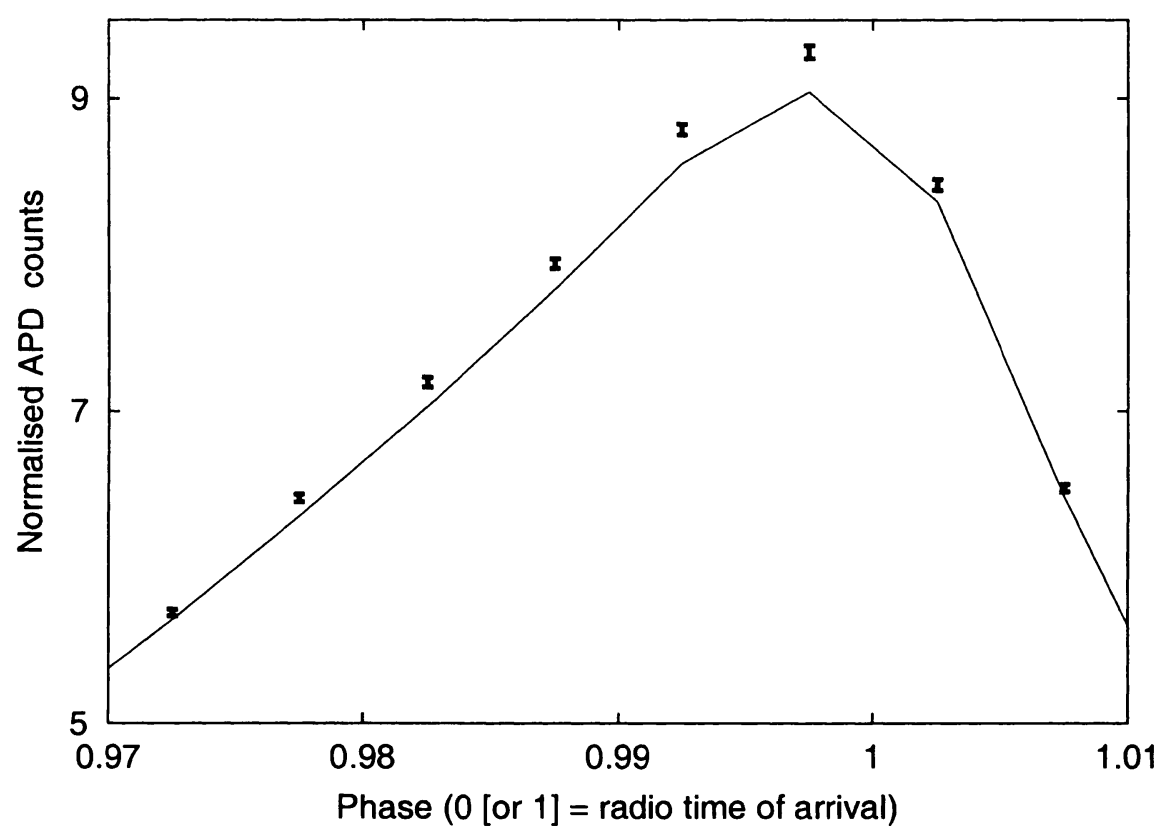

Figure 2. The mean optical "giant" pulse superimposed (with its error bars) on the average optical pulse. The average pulse is determined from the 40 pulsar periods centered on the GRP, but not including it, from both nights of observation.

Bank Crab Ephemeris (M. Roberts, private communication). After alignment, we found that the average GRP arrival time is $9.5 \mu$ s before that predicted by the JBE, well within the $19 \mu$ s error in this ephemeris. The average optical pulse arrived $100 \pm 20 \mu$ s before the JBE prediction (Fig. 1).

The radio data were searched for peaks which were more than 10- $\sigma$ above the noise level, which corresponded to giant pulses with a flux density of more than $150 \mathrm{Jy}$. Over the two nights of observations approximately 10000 such giant pulses were detected. Because the optical data were subject to fluctuations in sky transparency of $\sim 30 \mathrm{~s}$, only the photons that were detected 20 periods before and after the GRP, and during the GRP itself, were used for subsequent study. Folding the optical photons at the Crab's period and then averaging over all data sets (but not including the period associated with a GRP), we form an average pulse profile, as shown in Figure 2. For comparison we also show the pulse profile formed by averaging only the optical pulses coincident with a GRP. This profile shows that the giant optical pulses are on average $3 \%$ brighter than normal optical pulses. No statistically significant changes were found in other pulsar parameters such as arrival time, pulse shape, and interpulse height. We also find that the optical and GRP emission contain similar amounts of energy. 


\section{Conclusions}

Our observations that the optical emission from the Crab pulsar is enhanced when it is coincident with the presence of a GRP show for the first time that there is a clear link between the coherent and incoherent emission mechanisms. Along with recent positional coincidences of GRPs and X-ray emission (see Johnston \& Romani, these proceedings) it further strengthens the relationship between the high-energy emission location and the GRPs.

Of the possible mechanisms that might explain the radio-optical connection, we find one which provides the most consistent explanation. That is, that the enhanced optical emission is a reflection of an increase in the local plasma density which correspondingly causes the GRP event. This mechanism is attractive as it can explain why the large radio pulses correspond to quite small optical changes as the density enhancements, $n$, increase the coherent emission $\left(\propto n^{2}\right)$ with little effect on the (high-energy) incoherent radiation $(\propto n)$. These changes must occur on tiny timescales $(<10 \mu \mathrm{s})$ to explain the observed change in optical flux and the upper limit in the $\gamma$-ray region (Arons 1979). For further discussion please see Shearer et al. (2003).

Acknowledgments. We would like to thank our co-authors on this proceedings, O'Connor, Golden, Strom, Redfern, and Ryan, whose names couldn't appear above due to space restrictions.

\section{References}

Arons, J. 1979, Space Sci. Rev., 24, 437

Buckton, D., Ryan, O., Shearer, A., Redfern, R. M., \& Butler, R. F. 2003, Proc. SPIE, 4876, 1037

Harding, A. K., \& Daugherty, J. K. 1998, Adv. Sp. Res., 21, 251

Hegyi, D., Novick, R., \& Thaddeus, P. 1971, in IAU Symp. 46, The Crab Nebula, eds. R. D. Davies, \& F. G. Smith, (Dordrecht: Reidel), p. 129

Jones, D. H. P., Smith, F. G., \& Nelson, J. E. 1980, Nature, 283, 50

Lundgren, S. C., Cordes, J. M., Ulmer, M., Matz, S. M., Lomatch, S., Foster, R. S., \& Hankins, T. S. C. 1995, ApJ, 453, 433

Romani, R. W., \& Yadigaroglu, I.-A. 1995, ApJ, 438, 314

Romani, R. W. 1996, ApJ, 470, 469

Shearer, A., Stappers, B., O'Connor, P., Golden, A., Strom, R., Redfern, M., \& Ryan, O. 2003, Science, 401, 493

Voûte, J. L. L., Kouwenhoven, M. L. A., van Haren, P. C., Langerak, J. J., Stappers, B. W., Driesens, D., Ramachandran, R., \& Beijaard T. D. 2002, A\&A, 385, 733 